\title{
The TMT Instrumentation Program
}

\author{
Luc Simard $^{\mathrm{a}, \mathrm{b}}$, David Crampton ${ }^{\mathrm{a}, \mathrm{b}}$, Brent Ellerbroek ${ }^{\mathrm{a}}$, Corinne Boyer ${ }^{\mathrm{a}}$ \\ ${ }^{a}$ Thirty-Meter Telescope Project, 2632 E. Washington Blvd., Pasadena, CA, USA 91107 \\ ${ }^{b}$ National Research Council of Canada, Herzberg Institute of Astrophysics, 5071 West Saanich Rd., \\ Victoria, Canada, V9E 2E7
}

\begin{abstract}
An overview of the current status of the Thirty Meter Telescope (TMT) instrumentation program is presented. Conceptual designs for the three first light instruments (IRIS, WFOS and IRMS) are in progress, as well as feasibility studies of MIRES. Considerable effort is underway to understand the end-to-end performance of the complete telescopeadaptive optics-instrument system under realistic conditions on Mauna Kea. Highly efficient operation is being designed into the TMT system, based on a detailed investigation of the observation workflow to ensure very fast target acquisition and set up of all subsystems. Future TMT instruments will almost certainly involve contributions from institutions in many different locations in North America and partner nations. Coordinating and optimizing the design and construction of the instruments to ensure delivery of the best possible scientific capabilities is an interesting challenge. TMT welcomes involvement from all interested instrument teams.
\end{abstract}

Keywords: Extremely large telescopes, adaptive optics, science instruments, observation planning

\section{INTRODUCTION}

The TMT instrumentation program has made significant progress over the last two years on the instrument designs themselves as well as their end-to-end integration into the overall Observatory. This paper describes the science flowdown of requirements from the science cases to the instrument top-level requirements (Section 2), the early-light instrument designs (Section 3), observation workflows (Section 4) and future instrument development (Section 5).

\section{SCIENCE FLOWDOWN AND PROPOSED INSTRUMENTATION}

Science and instrumentation are obviously intimately linked, and it is important to establish the links between science cases and observatory requirements through a proper science flowdown ${ }^{1}$. TMT will provide insight into a wide range of astrophysical issues. For example, the TMT Detailed Science Case ${ }^{2}$ (DSC) describes forty-three science programs spanning a broad range of astrophysics. Reflective of the scientific and technical maturity of the TMT project, more than 230 scientific requirements have been captured in the TMT Science-based Requirements Document ${ }^{3}$ (SRD), and direct links between the science and the requirements have been established with a richly detailed Science Flowdown Matrix. Figure 1 is a high level schematic diagram of the flowdown from science to requirements.

The instrument suite that responds to the TMT scientific objectives is comprised of the nine capabilities listed in Table 1 plus the Alignment and Phasing System (APS) and the Near-IR Adaptive Optics System ${ }^{4}$ (NFIRAOS), and the associate Laser Guide Star Facility (LGSF). APS will use starlight to measure the wavefront errors and then will determine the appropriate commands to send to align the optics. NFIRAOS will feed diffraction limited images to IRIS and IRMS initially, and eventually to NIRES and WIRC. The instruments are located on two Nasmyth platforms with a maximum "payload" of 120 tons each, and an articulated tertiary mirror directs the telescope beam to the instrument stations. This configuration offers a number of key advantages: 1) multiple instruments can be addressed quickly with reduced overheads, 2) science with targets of opportunity and transient objects can be pursued, 3) throughput losses and thermal background are minimized, and 4) large and sensitive instruments operate in a gravity invariant, highly stable, and low vibration environment. Given the potentially significant impact of vibrations on AO performance, cooling solutions that minimizes vibrations such as pulse-tube coolers and closed-circuit, gaseous helium lines with remotely located compressors are being explored.

Ground-based and Airborne Instrumentation for Astronomy III, edited by lan S. McLean,

Suzanne K. Ramsay, Hideki Takami, Proc. of SPIE Vol. 7735, 773523 · C 2010

SPIE · CCC code: $0277-786 X / 10 / \$ 18 \cdot$ doi: $10.1117 / 12.857187$

Proc. of SPIE Vol. 7735 773523-1 


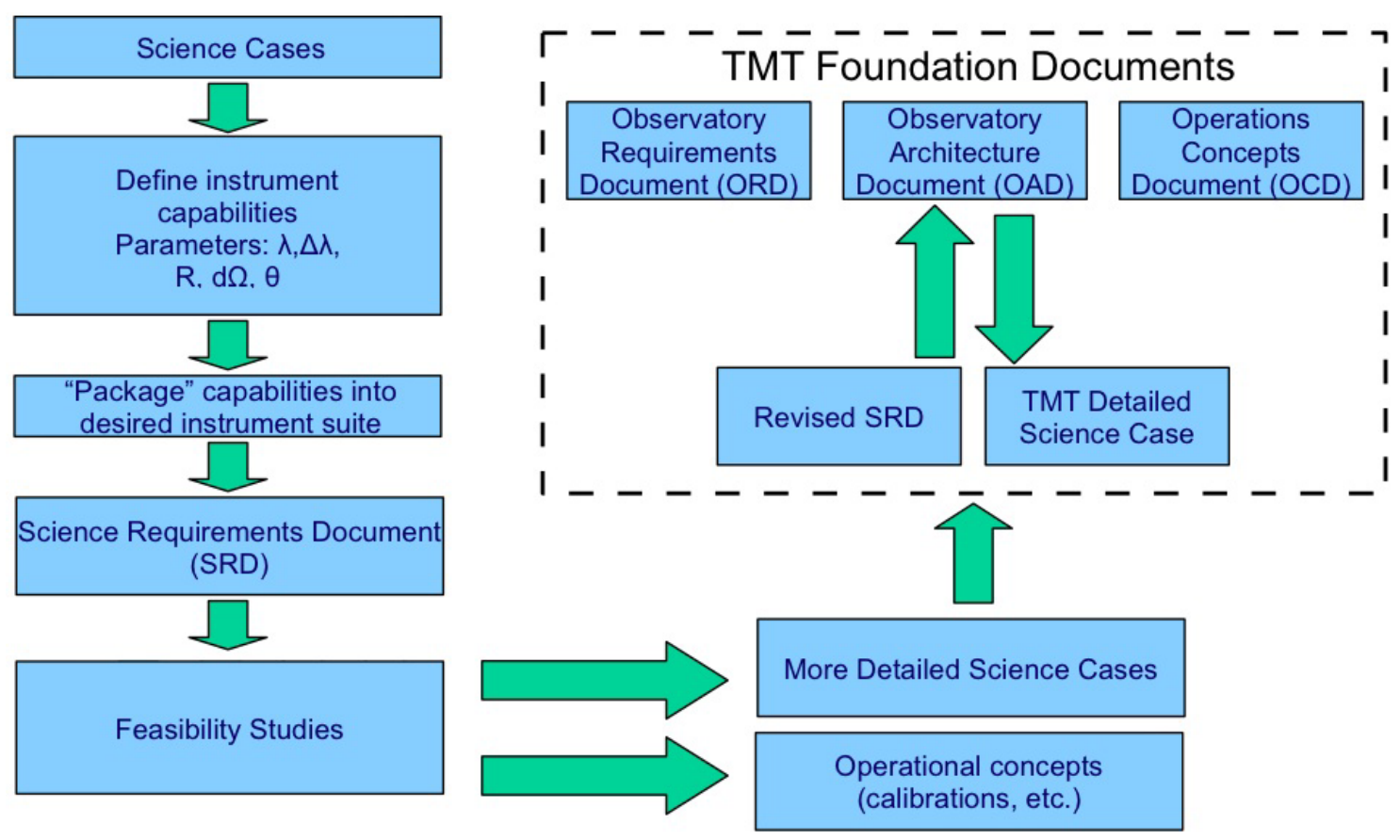

Figure 1. Schematic diagram of the TMT science flowdownt

Table 1. Instrument capabilities planned for the first decade of TMT operations. The early light instruments are highlighted in blue.

\begin{tabular}{|c|c|c|c|c|}
\hline Instrument & $\begin{array}{l}\text { Field of view / } \\
\text { slit length }\end{array}$ & Spectral resolution & $\lambda(\mu \mathrm{m})$ & Comments \\
\hline $\begin{array}{c}\text { InfraRed Imager and } \\
\text { Spectrometer } \\
\text { (IRIS) }\end{array}$ & $\begin{array}{c}<3^{\prime \prime} \text { IFU } \\
>15^{\prime \prime} \text { imaging }\end{array}$ & $\begin{array}{c}>3500 \\
5-100 \text { (imaging) }\end{array}$ & $\begin{array}{c}0.8-2.5 \\
0.6-5(\text { goal })\end{array}$ & NFIRAOS \\
\hline $\begin{array}{c}\text { Wide-field Optical } \\
\text { spectrometer and imager } \\
\text { (WFOS) }\end{array}$ & $\begin{array}{c}>40 \operatorname{arcmin}^{2} \\
>100 \operatorname{arcmin}^{2} \\
\quad(\text { goal }) \\
\text { Slit length }>500 "\end{array}$ & $\begin{array}{c}1000-5000 \\
>7500 @ 0.75 " \text { (goal) }\end{array}$ & $\begin{array}{c}0.31-1.0 \\
0.3-1.5(\text { goal })\end{array}$ & $\begin{array}{l}\text { Seeing- } \\
\text { Limited } \\
\quad(\mathrm{SL})\end{array}$ \\
\hline $\begin{array}{c}\text { InfraRed Multislit } \\
\text { Spectrometer (IRMS) } \\
\end{array}$ & $\begin{array}{c}2 \text { arcmin field } 46 \\
\text { deployable slits }\end{array}$ & $\begin{array}{c}\mathrm{R}=4660 @ 0.16 \\
\text { arcsec slit }\end{array}$ & $0.95-2.45$ & NFIRAOS \\
\hline $\begin{array}{c}\text { Multi-IFU imaging } \\
\text { spectrometer (IRMOS) }\end{array}$ & $\begin{array}{l}3 \prime \prime \text { IFUs over }>5 \\
\text { diameter field }\end{array}$ & $2000-10000$ & $0.8-2.5$ & MOAO \\
\hline $\begin{array}{l}\text { Mid-IR AO-fed Echelle } \\
\text { Spectrometer (MIRES) }\end{array}$ & $\begin{array}{l}3^{\prime \prime} \text { slit length } \\
10^{\prime \prime} \text { imaging }\end{array}$ & $5000-100000$ & $\begin{array}{c}8-18 \\
4.5-28(\text { goal }) \\
\end{array}$ & MIRAO \\
\hline $\begin{array}{l}\text { Planet Formation Instrument } \\
\qquad(\mathrm{PFI})\end{array}$ & $\begin{array}{l}1^{\prime \prime} \text { outer working } \\
\text { angle, } 0.05^{\prime \prime} \text { inner } \\
\text { working angle } \\
\end{array}$ & $\mathrm{R} \leq 100$ & $\begin{array}{c}1-2.5 \\
1-5 \text { (goal) }\end{array}$ & $\begin{array}{c}10^{8} \text { contrast } \\
10^{9} \text { goal }\end{array}$ \\
\hline $\begin{array}{l}\text { Near-IR AO-fed Echelle } \\
\text { Spectrometer (NIRES) }\end{array}$ & $2 "$ slit length & 20000-100000 & $1-5$ & NFIRAOS \\
\hline $\begin{array}{l}\text { High-Resolution Optical } \\
\text { Spectrometer (HROS) }\end{array}$ & $5^{\prime \prime}$ slit length & 50000 & $\begin{array}{c}0.31-1.1 \\
0.31-1.3 \text { (goal) } \\
\end{array}$ & SL \\
\hline $\begin{array}{l}\text { "Wide"-field AO imager } \\
\text { (WIRC) }\end{array}$ & $30^{\prime \prime}$ imaging field & $5-100$ & $\begin{array}{c}0.8-5.0 \\
0.6-5.0(\text { goal })\end{array}$ & NFIRAOS \\
\hline
\end{tabular}


Technical designs and operational concepts developed in 2005-2006 as part of the feasibility studies of the instrument capabilities listed in Table 1 provided the opportunity to explore the potential of TMT equipped with realistic instruments, and to verify and expand on the overall observatory requirements. After reviewing the instrument concepts, capabilities, and costs, the suite of science instruments was divided by the TMT Science Advisory Committee (SAC) in December 2006 into "early light" and "first decade" instruments. This selection was based on scientific priority and synergies with James Webb Space Telescope (JWST) and Atacama Large Millimeter Array (ALMA) as well as a variety of pragmatic reasons including funding constraints, commissioning practicalities, and technological readiness. The earlylight instruments are part of the TMT construction budget, and the remaining instruments will be developed, built and commissioned under the guidance of an instrumentation development office during the first decade of TMT operations. All instruments will be delivered with data reduction modules that can be used for quick-look pipelines and optimized by users to produce science-ready, processed data according to their specific science applications. More information on the proposed suite of TMT instruments is given in the TMT Instrumentation and Performance Handbook ${ }^{5}$.

\section{EARLY-LIGHT INSTRUMENTS}

\subsection{InfraRed Imaging Spectrometer (IRIS)}

The following organizations are involved in the IRIS science and technical teams: Caltech, UC (Los Angeles, Irvine, Santa Cruz, Berkeley), Herzberg Institute of Astrophysics, National Astronomical Observatory of Japan, and the University of Florida. The co-PIs are James Larkin (UCLA) and Anna Moore (Caltech). The project scientist is Betsy Barton (UC Irvine). A feasibility study was completed and externally reviewed in March 2006. A conceptual design study is currently underway and will be completed in October 2010. Figure 2 shows an exploded view of the full IRIS assembly.

IRIS is a diffraction-limited integral field spectrometer and imager designed to work with NFIRAOS. It includes a set of deployable wavefront sensors that feed tip-tilt-focus signals back to NFIRAOS. The imager has a field of view of 17"x17" and deliver images with total wavefront errors under $30 \mathrm{~nm}$. The spectrometer will take advantage of two slicing techniques that are suited to different types of science programs. A lenslet channel will maximize image quality whereas a slicer channel will maximize sensitivity. The lenslet will be used to observe at the finest plate scales. It is easy to expand spatially to $>100 \times 100$ to sample the entire point-spread-function (PSF) even at 4 milliarcsec scale. It also has intrinsically low wavefront error because lenslets sample the image plane. A slicer will be used to observe at coarser plate scale. Wavelength coverage can be easily expanded once a sufficiently large field is achieved at coarse scale. Both IFU channels will share a common set of foreoptics (collimator, filters, rotating pupil mask and crossed Ameci atmospheric dispersion corrector). The selection between the lenslet and slicer paths will be done with simple twoposition stages, and the beams will share a common path at the grating. Following the grating, both channels will make use of the same camera and detector. 


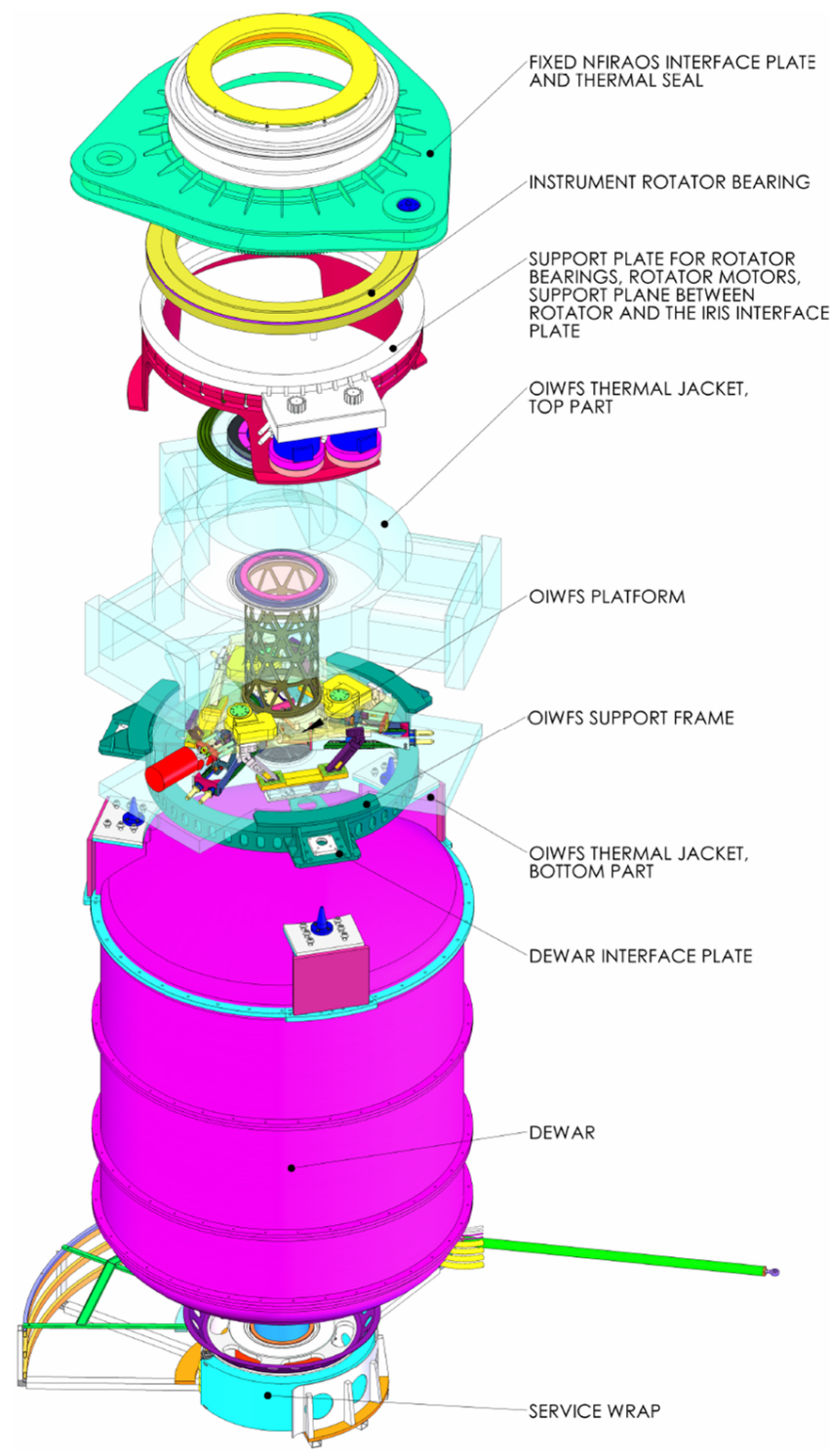

Figure 2. Exploded view of the IRIS Assembly. This assembly is mounted on the bottom port of the NFIRAOS AO system. The assembly includes three main subsystems from top to botton: the instrument rotator, the on-instrument wavefront sensors and the science dewar.

The reference design of NFIRAOS includes one or more tip/tilt/focus natural guide star wavefront sensors within each instrument (Figure 3). NFIRAOS will sharpen the images of natural guide stars and improve the sky coverage for tip/tilt sensing with these on-instrument wavefront sensors (OIWFS).

To maximize sky coverage, these on-instrument wavefront sensors will sense natural guide stars in the infrared. For the IRIS science instrument, two tip/tilt wavefront sensors will be used to perform fast guiding, and one tip/tilt/focus wavefront sensor will be used to calibrate the focus biases in the Laser Guide Star WFS induced by the variations in the range to the mesospheric sodium layer. All three OIWFS will have the dual tip-tilt/tip-tilt-focus functionality to maximize sky coverage. The IRIS OIWFS will also enable computation of blind modes undetectable using the laser guide star wavefront sensors in NFIRAOS, comprised of a mix of focus/astigmatism and tilt anisoplanatism modes. Control of these modes is important for optimizing astrometric accuracy and image quality over non-zero fields of view. 


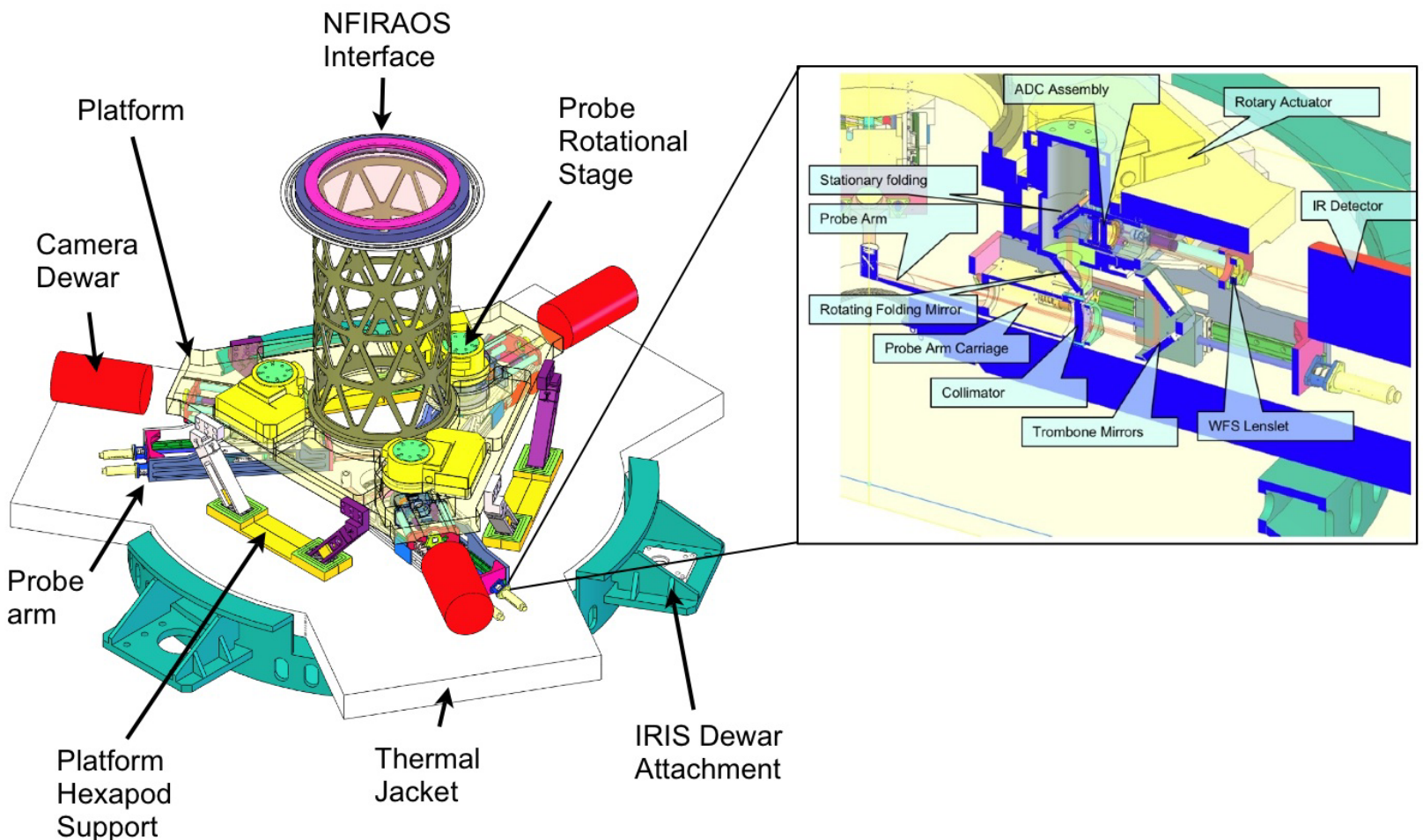

Figure 3. IRIS on-instrument wavefront sensor subsystem. The inset shows the detailed opto-mechanical design of one of the probe arms.

More details on IRIS are given in a series of other papers ${ }^{6,7,8,9,10,11}$ included in these proceedings. The IRIS website is at http://irlab.astro.ucla.edu.

\subsection{Wide-Field Optical Spectrometer (WFOS)}

WFOS is a collaboration between Caltech and UC Santa Cruz. The principal investigator is Rebecca Bernstein (UCSC), the project manager is Bruce Bigelow (UCSC), and the project scientist is Charles Steidel (Caltech).

The current concept for WFOS is the Multi-Object Broadband Imaging Echellette (MOBIE) spectrometer. The MOBIE conceptual design study is underway. MOBIE aims to provide flexibility in terms of resolution and multiplexing, as well as complete wavelength coverage at all resolutions while minimizing mechanical complexity.

MOBIE is an imaging spectrograph designed to perform multi-object spectroscopy, single object spectroscopy, or direct imaging, of very faint sources throughout the optical waveband $(0.31-1.0 \mu \mathrm{m})$ using seeing-limited images delivered by TMT. It is designed to have very high throughput ( $>30 \%$ at all wavelengths, from slit to detector) in order to preserve the aperture advantage of TMT relative to current-generation multi-object spectrographs on 8m-class telescopes. MOBIE will be equipped with two guider/wavefront sensing cameras placed just outside the instrument field of view, as well as a deployable slit-viewing camera to be used for single-object observations or as an additional option for guiding/wavefront sensing. It is envisioned that one of these cameras will be used for guiding, and another for maintaining telescope focus using low-order wavefront sensing signals, and will communicate with the telescope control system and the secondary mirror control system, respectively.

MOBIE will include an atmospheric dispersion corrector (ADC) that adjusts automatically to account for zenith distance of the telescope pointing to ensure that the positions of targets are achromatic over the 0.31-1.0 $\mu \mathrm{m}$ wavelength range. The instrument will include a closed-loop flexure compensation system that removes any instrument-rotator-dependent flexure at the required level to maintain image quality and the effectiveness of calibrations. 
The MOBIE optical design makes use of a field centered at a position 5.4 arcmin from the telescope optical axis in order to allow for the use of a reflective paraboloidal (achromatic) collimator that functions over the whole design wavelength of the instrument. The $300 \mathrm{~mm}$ collimated beam is then separated into blue $(0.31-0.55 \mu \mathrm{m})$ and red $(0.55-1.0 \mu \mathrm{m})$ channels using a dichroic beam-splitter, forming two separate beams optimized over these wavelength ranges, with separate dispersers, filters, cameras, and detectors. An illustration of the MOBIE optical layout is given in Figure 4.

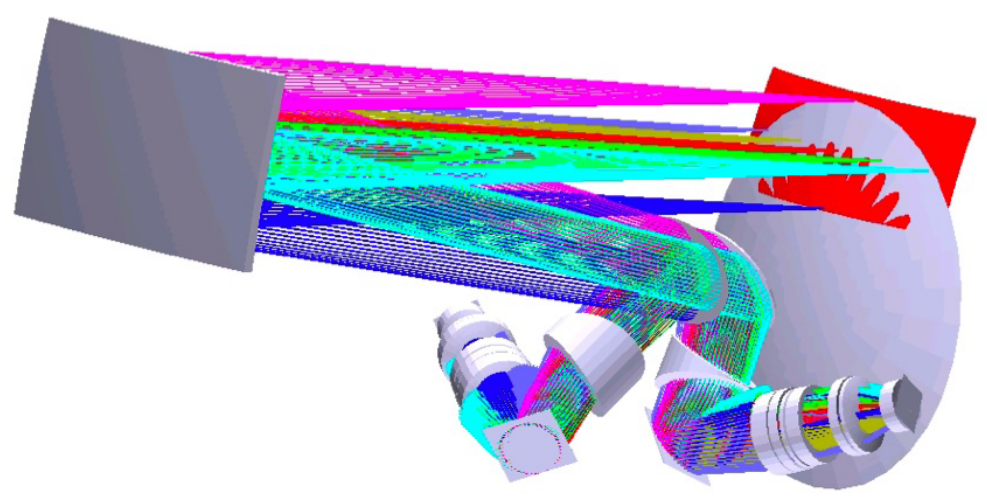

Figure 4. Optical layout of MOBIE. Rays arrive at the focal surface (first grey surface on the right) from the right hand side. The red rectangle indicates rectangular field of view of the baseline MOBIE reflecting collimator. Rays continue to the collimating mirror on the left, and back toward the dichroic and red fold mirror. The red and blue arms are shown here on opposite sides of the optical axis for symmetry.

MOBIE will provide a highly versatile range of spectral resolution and multiplexing capability through the use of conventional ruled reflection gratings on each wavelength channel of the instrument. In the lowest-dispersion mode $(\mathrm{R}=1000)$ the necessary wavelength coverage is obtained with a single spectral order for each wavelength channel, so that simultaneous spectroscopic observation of $>100$ targets within the MOBIE field of view will be possible with complete wavelength coverage. In higher resolution modes, a different reflection grating, together with a crossdispersing prism, is used to obtain complete wavelength coverage on each spectral channel in a single exposure of $20-$ 40 objects in an "echellette" format (ECH), where the number of targets which can be observed simultaneously depends on the number of spectral orders needed. At a given spectral resolution, smaller wavelength coverage and greater multiplexing is accommodated through the use of order-sorting filters in the beam of each spectral channel, to select one or more orders of interest. If only a single spectral order is needed on each channel (i.e., no cross-dispersion is needed), then slits of arbitrary length up to the full field length of 9.6 arcmin may be used. Thus, the astronomer can select whatever combination of spectral coverage and multiplexing is required by the science, at a given spectral resolution. MOBIE will incorporate a robust structure to support the various components of the instrument and minimize instrument flexure. In addition, it will incorporate an enclosure to protect its components and provide a light tight environment for the optical elements. The basic design of the support structure and enclosure will also offer ready access to all configurable portions of the instrument, such as the ADC, slit masks, dichroics, filters, gratings, and detectors to facilitate set-up and maintenance. MOBIE is being designed so as not to preclude additional functionality such as an integral field unit, a tunable filter for narrow-band imaging, and an interface to a possible future Ground-Layer Adaptive Optics system for improving the images delivered to the instrument, and thus allowing for higher-sensitivity observations.

The MOBIE concept builds on many successful design features that were pioneered on the most recent generation of instruments for the $6.5-10 \mathrm{~m}$ class telescopes. For example, the baseline collimator design for MOBIE, which uses an off-axis parabolic reflector addressing a rectangular off-axis field, was first developed for the Keck LRIS imaging spectrograph, and subsequently used on the Keck DEIMOS and ESI instruments as well. The concept of a multi-object echellette spectrograph format was first demonstrated on the IMACS imaging spectrograph for the Magellan telescopes. The utility and efficiency of splitting the instrument optical path into red and blue color channels, separated by a dichroic, has been used by many other existing spectrographs, including LRIS on Keck, UVES on the VLT, MIKE on Magellan, MODS on the LBT, and many others. Thus, while MOBIE is a new and unique instrument design, not only 
for TMT, but in the world of optical spectrographs, it is also a system composed of many familiar and proven building blocks.

More details on the design of MOBIE are given elsewhere ${ }^{12,13}$ in these proceedings.

\subsection{InfraRed Multi-slit Spectrometer (IRMS)}

Some form of multi-object, near-infrared spectroscopy is another essential capability for early light. Understanding the so-called "First Light" objects in the Universe, the origin and evolution of galaxies and other objects detected by JWST and ALMA will require spectra of many extremely faint objects in the NIR, and multiplexing will thus be essential ${ }^{14}$. Although SAC advocated a fully multiplexed deployable IFU system using Multiple Object AO (MOAO), this was judged to be too risky and expensive for an early light instrument. Fortuitously a clone of the MOSFIRE multislit instrument, currently being built for Keck, provides a very exciting interim capability. Although MOSFIRE will be a seeing-limited instrument for Keck, it can be easily adapted for use in an AO mode with NFIRAOS, providing an exceedingly powerful capability for TMT at low risk and modest cost. When optimized for wide-field mode, NFIRAOS will deliver images to IRMS that will produce almost an order of magnitude gain in encircled energy within narrow (160 milliarcsecs) slits over the entire of 2' diameter field. NFIRAOS+IRMS is expected to deliver a K-band encircled energy within a radius of 80 milliarcsecs six times higher than for seeing limited observations (Figure 5).
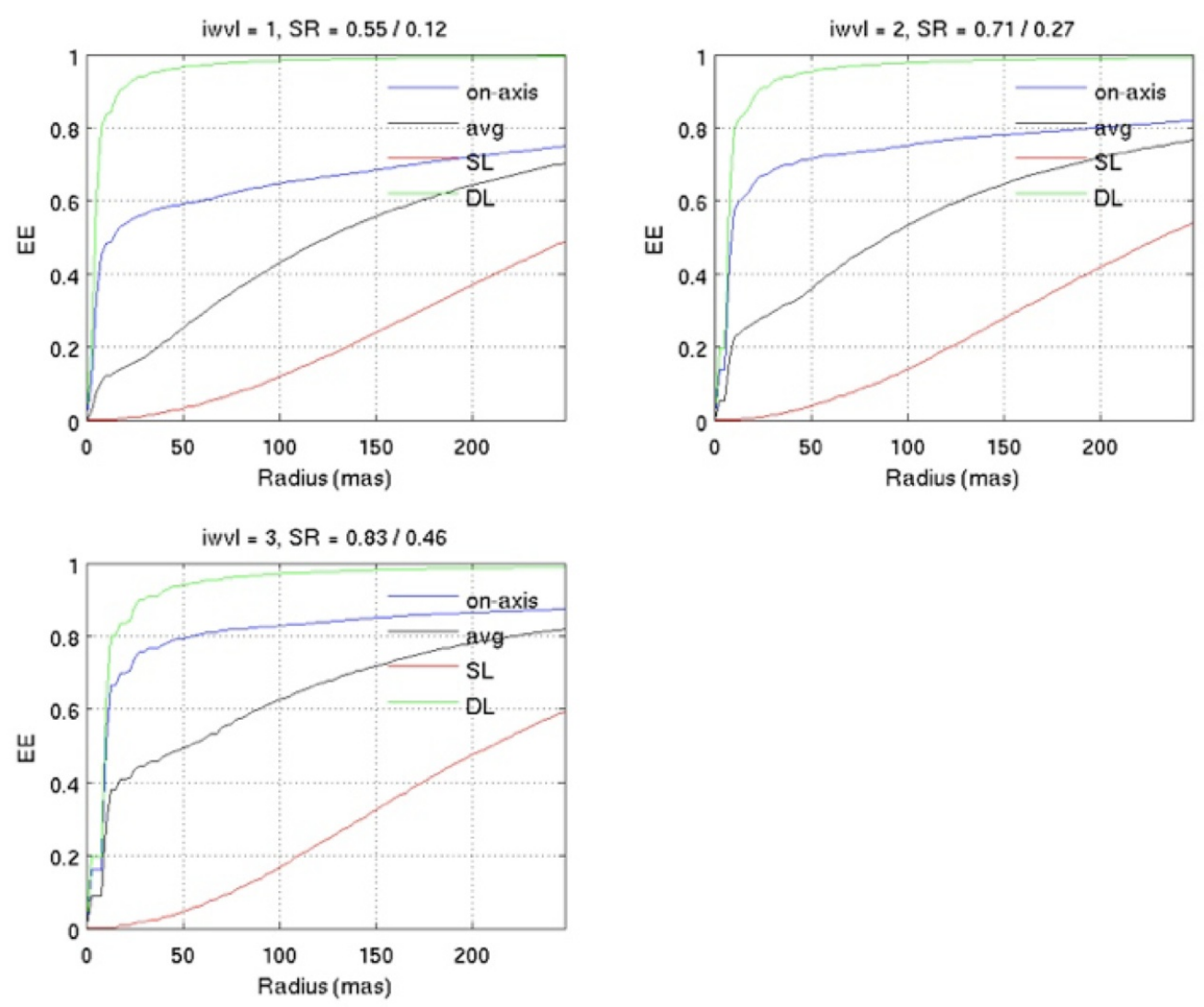

Figure 5. Encircled energy curves showing the NFIRAOS+IRMS wide field performance in J (top left), H (top right) and K (bottom left) with tomographic reconstructor parameters tuned to optimize image quality over the full NFIRAOS field of view (120" in diameter). The minimum (Nyquist-sampled) IRMS slit width is 160 milliarcsecs, and it thus encloses light within a radius of 80 milliarcsecs. The different curves shown are diffraction-limited (green), on-axis (blue), average (black) and seeing-limited (red). 
MOSFIRE is a multi-slit instrument designed for the $\mathrm{f} / 15$ Cassegrain focus on the Keck 1 telescope. This is the same $\mathrm{f} /$ ratio as for the TMT Nasmyth focus and without change to the MOSFIRE design, it would naturally take in the entirety of the NFIRAOS field of regard of 2' in diameter.

MOSFIRE uses a cryogenic slit mask unit (CSU) that was originally developed as a back up for the JWST NIRSPEC instrument. The spatial pixel scale of IRMS on TMT is reasonably well matched to the sampling scale (50 milliarcsecs) requested in the SRD for IRMOS and the length of individual slitlets (made by masking bars in MOSFIRE) is similar to the recommended scale for IRMOS. For multiplexing, the individual bars can be configured in up to 46 slitlets over the entire NFIRAOS field; in practice, some of the slitlets would be made into contiguous slits of lengths that are multiples of $2^{\prime \prime} .4$. The width of slits and their placement within the field are remotely configurable in real time.

IRMS on TMT will also offer additional capabilities. It can be configured as a long slit spectrograph or as an imager that would cover the entire NFIRAOS field of regard, albeit with spatial sampling of only 33-60 milliarcsecs (roughly 2-4 times larger than the diffraction limit at 2 microns). It could also be used in a seeing-limited mode (for either imaging or spectroscopy), if desired, by flattening the deformable mirrors in NFIRAOS and turning off the AO correction.

Details on the MOSFIRE project can be found at http://www.astro.ucla.edu/ irlab/mosfire/.

\section{OBSERVATION WORKFLOWS}

The TMT project has done a detailed study of the workflows that will be followed to conduct observations at the telescope. The goal of this exercise was to ensure that acquisition and calibration requirements would be met.

A TMT science observation consists of a hierarchical series of linked processes. The first phase of the observation is the Observation Preparation. The output of this phase includes all of the information necessary to perform the observation, in particular the coordinates of the science object, the coordinates of the natural guide stars, their magnitudes and color temperature, etc. The second phase of the Observation is Acquisition, which consists of slewing the telescope to the science target, configuring the AO system and instrument, acquiring the laser and natural guide stars and closing the adaptive (or active) optics loops. An example of this phase is described in details in Section 4.4 below. Once the acquisition process is completed, the next process is the Observation Execution. During this phase, raw science data and required nighttime calibrations are acquired. Finally, Post Observations Activities include PSF reconstruction, pipeline data reduction, and data archiving.

\subsection{Top-level requirements}

The top-level requirements for the observation preparation, the observation acquisition, the observation execution and the post observation activities are defined in the Observatory Requirement Document ${ }^{15}$ (ORD) and in the Operations Concept Document ${ }^{16}(\mathrm{OCD})$.

In particular, the following requirements have been defined for the acquisition phase and are summarized here:

- The telescope and enclosure shall be able to point from any one position on the sky to any other within 3 minutes (REQ-1-ORD-1800)

- During steady-state science operations, the system shall be capable of completing the target acquisition sequence in 5 minutes if an instrument change or a major instrument reconfiguration is not needed (REQ-1ORD-1805).

- In the case of an instrument change or a major instrument reconfiguration, the maximum time to complete the target acquisition sequence is 10 minutes (REQ-1-ORD-1810 and REQ-1-ORD-1815).

The acquisition sequences studied as part of this exercise demonstrate that the TMT systems will be flexible enough to accommodate a diversity of acquisition scenarios. This flexibility should be adequate to tackle more complex sequences not studied yet that some programs will need. However, it should be emphasized that added complexity will lead to greater risk of exceeding the 5 minute time budget.

\subsection{Guidestar catalogs and astrometry assumptions}

The efficiency of the acquisition sequences will obviously depend on how well target fields have been characterized prior to TMT observations. In particular, the availability of precise astrometric solutions measured from high quality 
imaging and/or catalogs will be of prime importance to determine the relative positions of suitable natural guide stars relative to the science target. However, this will not always be possible, especially for targets of opportunity such as gamma-ray bursts and other transient phenomena that may occur in previously unobserved regions of the sky. Acquisition sequences for Target of Opportunity ( $\mathrm{ToO}$ ) may therefore include additional steps and may require more time to complete. Large surveys covering most of the sky (i.e., VISTA) may alleviate this problem.

For the acquisition processes described below, prior knowledge of the target fields is assumed to include the following:

- Objects suitable as natural guide stars. Extended objects should not be included in this list.

- Astrometric solutions applicable at the wavelength of the wavefront sensors. For instruments such as IRIS and IRMS, astrometric solutions would be obtained from near-IR observations whereas WFOS will require astrometric solutions obtained at optical wavelengths.

\subsection{Inputs to the observation execution}

Observing programs will need to provide the following inputs for the successful execution of the observations:

- Coordinates of science object/field

- Rate of apparent motion for non-sidereal targets

- Positions, magnitudes and colors of natural guide stars

- Instrument configuration

- Sequence of science and nighttime calibration exposures (arc, flats, darks, off-source)

- Type and sequence of daytime calibrations

- Spectrophotometric standard star observations

- Radial velocity standard star (if needed)

- Weather conditions under which observations should be ideally conducted.

This list is a minimum set, and additional observation input parameters may be required. It is also important to note that these inputs will need to be provided by observers in a very detailed format that can be stored in an observing database so that observing programs can be efficiently sequenced. Sequencing will be key in meeting the required acquisition time budgets.

\subsection{An example: Laser-guide star adaptive optics and IRIS IFU mode}

Figure 6 shows the acquisition sequence for an observation using the IRIS integral spectroscopy unit assisted by laser guide star adaptive optics. The baseline acquisition scenario uses the NFIRAOS acquisition camera to locate the guide stars after the telescope slew. However, the field of view of the three on-instrument wavefront sensors will be large enough to allow direct guide star acquisition using only these wavefront sensors once the telescope pointing model has had time to mature to meet its accuracy requirement. Timing estimates for the steps in this acquisition sequence add up to less than 300 seconds. This is not unreasonable given that well-sequenced telescopes such as Gemini can already switch from a seeing-limited, longslit observation to a full AO-assisted IFU one in just a few minutes.

\section{FUTURE INSTRUMENT DEVELOPMENT}

The development of new, powerful instruments is obviously essential to maintaining TMT at the forefront of international astronomical research. It is driven by the recognition that capabilities similar to the first decade instrument suite shown in Table 1 are required to fulfill TMT science mission, and that these capabilities will be needed as soon as possible after early light. It is important to emphasize that "future instruments" here includes both AO systems and science instruments.

\subsection{A Global Partnership}

There is strong interest from all TMT partners in participating in instrument projects. This is driven primarily by the scientific interests of their respective science communities. The emerging TMT partnership involves countries and 
institutions that are distributed over large geographical distances and that are used to different development models. They bring a broad range of facilities and capabilities to the TMT instrumentation effort. The potential is exciting, and significant efforts are already under way to achieve the goal of building instrument partnerships that make sense scientifically and technically while satisfying partner aspirations.

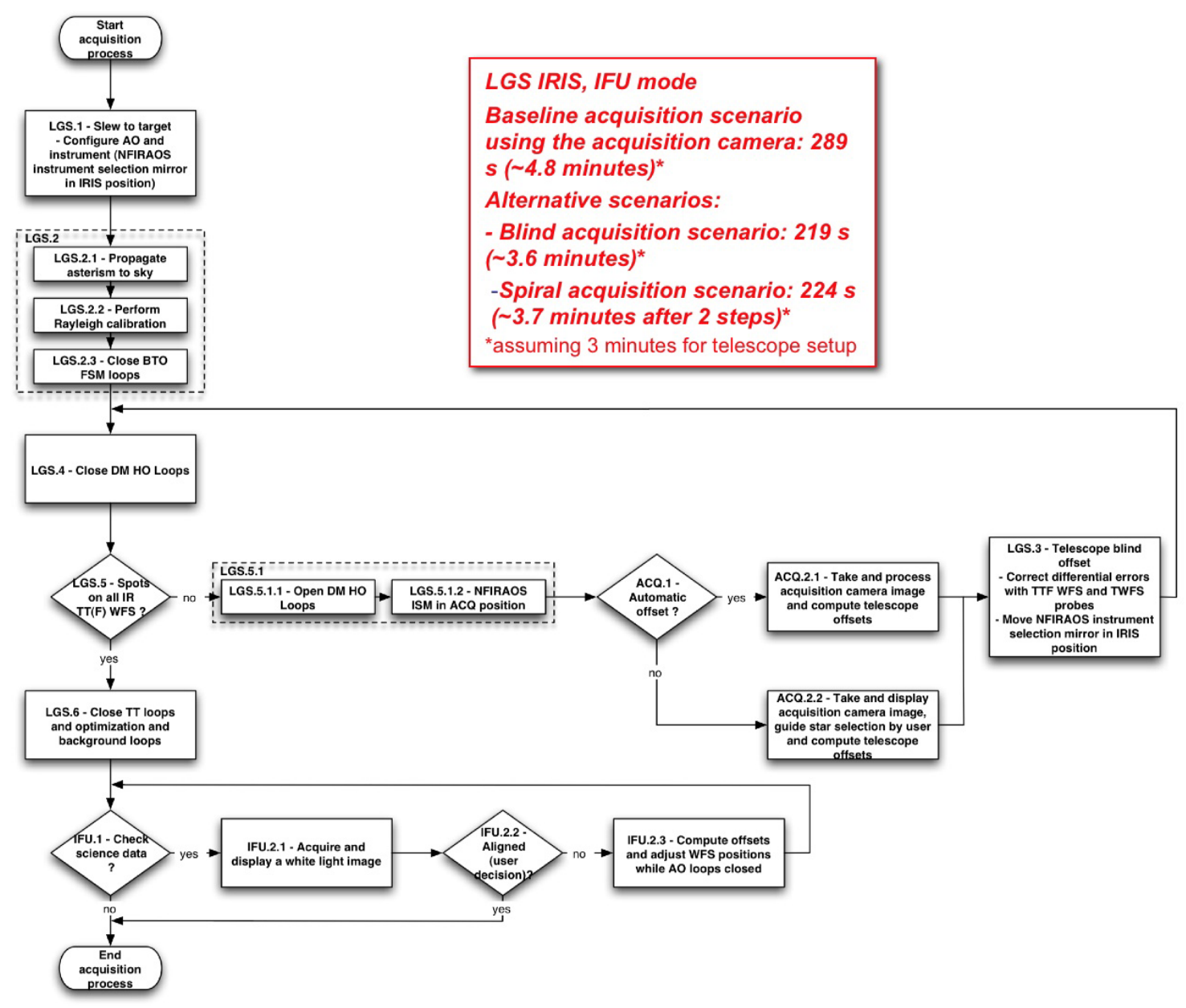

Figure 6. Acquisition sequence for the IRIS IFU with laser-guide star adaptive optics.

Beyond the current work on the early-light instrument suites, a number of teams are looking over existing and new designs for the next generation of instruments. It is important to emphasize that the instrument concepts shown in Table 1 may or may not be the ones that will actually be built. These concepts were studied at the feasibility level back in 2005-2006, and new ideas are certain to emerge from regular reassessments of scientific priorities by the Scientific Advisory Committee. For example, a team is currently revisiting the concept for a mid-infrared Echelle spectrometer (MIRES) on $\mathrm{TMT}^{17,18}$. The new design will include imaging and low spectral resolution modes.

\subsection{Instrument Development Office}

Future instrument development will be the responsibility of the TMT Instrument Development Office (IDO). This is a joint $\mathrm{AO}$ and science instrumentation engineering team that will provide oversight for all instrumentation activities (except routine support). It will play a central role within the diverse TMT partnership. The IDO will initially be primarily occupied with the early-light instruments (WFOS, IRIS, IRMS, NFIRAOS) and associated AO systems with an increasing shift of effort towards support for future instruments and AO systems. 
The IDO will have its own annual development fund, and this fund will be kept separate from observatory operations. It will be used for building some of the more modest instruments in the planned suite and/or for funding the earlier development stages of the more ambitious, more complex ones.

\subsection{Development Process}

The prioritization of future TMT instruments must be driven by scientific objectives. This is the cornerstone of the development program, and it will therefore be the responsibility of the TMT SAC. However, prioritization is not only a matter of science. All available information on technical readiness, schedule and cost will also enter instrument decisions made by the SAC.

The development process is expected to include the following steps:

- Extensive consultation with the broader TMT user community

- SAC discussions of instrument options and requirements based on desired science, technical readiness, schedule and cost

- $\quad \mathrm{SAC}$ prioritizes the next few instruments and makes recommendations to the TMT Board

- Board establishes guidelines (including scope and cost targets) for studies

- Two one-year competitive conceptual designs are initiated for each instrument

- SAC makes recommendations based on the outcome of studies (scientific capability, priorities, options, etc.)

- The Observatory (and Board) will negotiate cost and scope of instrument contract awards taking partnership issues into consideration.

- $\quad$ TMT will provide project management and oversight in all instruments:

- To ensure compatibility with overall Observatory system

- To maximize operational efficiency, reliability and minimize cost

$\circ$ To encourage common components and strategies

○ To ensure that budgets and schedules are respected

\section{ACKNOWLEDGEMENTS}

The authors gratefully acknowledge the support of the TMT partner institutions. They are the Association of Canadian Universities for Research in Astronomy (ACURA), the California Institute of Technology and the University of California. This work was supported as well by the Gordon and Betty Moore Foundation, the Canada Foundation for Innovation, the Ontario Ministry of Research and Innovation, the National Research Council of Canada, the Natural Sciences and Engineering Research Council of Canada, the British Columbia Knowledge Development Fund, the Association of Universities for Research in Astronomy (AURA) and the U.S. National Science Foundation.

\section{REFERENCES}

[1] Simard, L. and Crampton, D., "Science Flowdown for the Thirty Meter Telescope," in [Ground-based and Airborne Instrumentation for Astronomy III], McLean, I. S., Ramsay, S. K., and Takami, H., eds., Proc. SPIE 7735-213 (2010).

[2] TMT Detailed Science Case: 2007 (www.tmt.org/sites/default/files/TMT-DSC-2007-R1.pdf)

[3] TMT Science-based Requirements Document (SRD, www.tmt.org/sites/default/files/SRD-CCR18-Science-BasedRequirements-Document.pdf)

[4] Herriot, G. et al., "NFIRAOS: Facility Adaptive Optics System for the TMT," in [Adaptive Optics Systems II], Ellerbroek, B. L., Hart, M., Hubin, N., and Wizinowich, P. L., eds., Proc SPIE 7736-9 (2010) 
[5] TMT Instrumentation and Performance Handbook (www.tmt.org/sites/default/files/TMT-Instrumentation-andPerformance-Handbook.pdf)

[6] Larkin, J. E., Moore, A. M., Barton, E. J., Bauman, B. J., Bui, K., Canfield, J. M., Crampton, D., Delacroix, A., Fletcher, J. M., Hale, D. D. S., Niehaus, D. L. C. N., Phillips, A. C., Reshetov, V. A., Simard, L., Smith, R. M., Suzuki, R., Usuda, T., and Wright, S. A., "The infrared imaging spectrograph (IRIS) for TMT: instrument overview," in [Ground-based and Airborne Instrumentation for Astronomy III], McLean, I. S., Ramsay, S. K., and Takami, H., eds., Proc. SPIE 7735-79 (2010).

[7] Moore, A. M., Bauman, B. J., Barton, E. J., Crampton, D., Delacroix, A., Larkin, J. E., Simard, L., and Wright, S. A., "The infrared imaging spectrograph (IRIS) for TMT: spectrograph design," in [Ground-based and Airborne Instrumentation for Astronomy III], McLean, I. S., Ramsay, S. K., and Takami, H., eds., Proc. SPIE 7735-87 (2010).

[8] Barton, E. J., Larkin, J. E., Moore, A. M., Wright, S. A., Crampton, D., and Simard, L., "The infrared imaging spectrograph (IRIS) for TMT: the science case," in [Ground-based and Airborne Instrumentation for Astronomy III], McLean, I. S., Ramsay, S. K., and Takami, H., eds., Proc. SPIE 7735-208 (2010).

[9] Wright, S. A., Barton, E. J., Larkin, J. E., Moore, A. M., Crampton, D., and Simard, L., "The infrared imaging spectrograph (IRIS) for TMT: sensitivities and simulations," in [Ground-based and Airborne In- strumentation for Astronomy III], McLean, I. S., Ramsay, S. K., and Takami, H., eds., Proc. SPIE 7735-284 (2010).

[10]Phillips, A. C., Bauman, B. J., Larkin, J. E., Moore, A. M., Niehaus, C. N., Crampton, D., and Simard, L., "The infrared imaging spectrograph (IRIS) for TMT: the atmospheric dispersion corrector," in [Ground-based and Airborne Instrumentation for Astronomy III], McLean, I. S., Ramsay, S. K., and Takami, H., eds., Proc. SPIE 7735212 (2010).

[11]Loop, D., Reshetov, V. A., Fletcher, J. M., Wooff, R., Dunn, J., Moore, A. M., Smith, R. M., Hale, D. D. S., Dekany, R. G., Wang, L., Ellerbroek, B. L., Simard, L., and Crampton, D., "TMT infrared imaging spectrograph (IRIS): wavefront sensing and AO interface, " in [Ground-based and Airborne Instrumentation for Astronomy III], McLean, I. S., Ramsay, S. K., and Takami, H., eds., Proc. SPIE 7735-285 (2010).

[12] Bernstein, R. A. and Bigelow, B. C., "Progress of the conceptual design for the MOBIE imaging spectrograph for TMT," in [Ground-based and Airborne Instrumentation for Astronomy III], McLean, I. S., Ramsay, S. K., and Takami, H., eds., Proc. SPIE 7735-77 (2010).

[13]Ellis, K. S., Bernstein, R. A., and Bigelow, B. C., "Conceptual design phase stray light of the MOBIE imaging spectrograph for TMT," in [Ground-based and Airborne Instrumentation for Astronomy III], McLean, I. S., Ramsay, S. K., and Takami, H., eds., Proc. SPIE 7735-215 (2010).

[14] Mobasher, B., Weber, B., Crampton, D., Simard, L., and Konidaris, N., "An infrared multi-object spectrograph (IRMS) with adaptive optics for TMT: the science case," in [Ground-based and Airborne Instrumentation for Astronomy III], McLean, I. S., Ramsay, S. K., and Takami, H., eds., Proc. SPIE 7735-211 (2010).

[15] TMT Observatory Requirements Document (ORD, www.tmt.org/sites/default/files/documents/application/pdf/ordccr22.pdf.pdf)

[16] TMT Operations Concept Document (OCD, www.tmt.org/sites/default/files/documents/application/pdf/ocdccr08.pdf)

[17] Okamoto, Y.K. et al., "The science drivers for a mid-infrared instrument for the TMT," in [Ground-based and Airborne Instrumentation for Astronomy III], McLean, I. S., Ramsay, S. K., and Takami, H., eds., Proc. SPIE 7735210 (2010).

[18] Tokunaga, A. T. et al., "The design of mid-infrared instrument for the Thirty Meter Telescope," in [Ground-based and Airborne Instrumentation for Astronomy III], McLean, I. S., Ramsay, S. K., and Takami, H., eds., Proc. SPIE 7735-82 (2010). 